\title{
Analysis of Pitting Corrosion in Aerospace Aluminium Alloy AA2024-T3
}

\author{
A.P. Boag ${ }^{*}$, D.G. McCulloch ${ }^{*}$, D.N. Jamieson ${ }^{* *}$, A.E. Hughes ${ }^{* * *}$, L.M. Pedrina ${ }^{*}$ and C.G. Ryan ${ }^{* * * *}$ \\ * Applied Physics, School of Applied Science, RMIT University, GPO Box 2476V, Melbourne, \\ VIC, 3001, Australia \\ ** Microanalytical Research Centre, School of Physics, University of Melbourne, Victoria, 3010, \\ Australia \\ *** CSIRO, Division of Manufacturing and Infrastructure Technology, Private Bag 33 Clayton Sth \\ MDC, Clayton, Vic, 3169, Australia \\ **** CSIRO, Division of Exploration and Mining, Bayview Avenue, Clayton, VIC 3168, Australia
}

\section{Introduction}

Aluminium alloys for commercial applications contain numerous intermetallic inclusions [1]. Intermetallics can have diameters up to $50 \mu \mathrm{m}$ [2] and particle surface densities ranging from $3 \times 10^{5}$ to $1 \times 10^{6} / \mathrm{cm}^{2}$ on a rolled surface [2-4]. Exposed surfaces of these alloys require protection against corrosion. This is usually accomplished by means of a conversion coating using $\mathrm{Cr}$ compounds or anodising, followed by a paint system which may include a chromate inhibited primer and a topcoat. The onset of corrosion in the aluminium alloy occurs when these coatings breakdown, this is a significant problem given the wide application of aluminium alloys in infrastructure and transport.

Pitting corrosion in aluminium alloys is particularly destructive since pitting can lead to other forms of corrosion, such as exfoliation corrosion which undermines structural integrity [5]. Pitting corrosion is localised and it has been demonstrated that intermetallics play an important role in the formation of a pit $[3,4]$. This study will utilise a range of microscopic and electrochemical techniques to determine both physically and statistically the association of intermetallics with the onset of deep pits in a corroded surface.

\section{The Alloy Samples}

Polished Al-alloy AA2024-T3 [5] substrates are prepared by pressing out discs $1.6 \mathrm{~mm}$ thick. The main alloying elements are $\mathrm{Cu}(3.8-4.9 \%)$ and $\mathrm{Mg}(1.2-1.8 \%)$. It also contains $\mathrm{Si}(0.5 \%), \mathrm{Fe}(0.5 \%)$, $\mathrm{Mn}(0.3-0.9 \%), \mathrm{Cr}(0.1 \%), \mathrm{Zn}(0.25 \%)$ and $\mathrm{Ti}(0.15 \%)$. These discs will then be ground using silicon carbide papers to $\mathrm{P} 1200$, before final polishing down with $0.25 \mu \mathrm{m}$ diamond paste. Corrosion was initiated by placing samples in a $0.5 \mathrm{M} \mathrm{NaCl}$ solution, until corrosion in the form of pits is identified by hydrogen evolution at the surface and an increase in the corrosion current.

\section{Electrochemical Analysis}

While corroding the surface of the alloy sample the corrosion current and potential will be continuously monitored. By monitoring these electrochemical parameters the onset of localised corrosion as well as the corrosion rate will be determined. An optical microscope will also be used to view the corrosion process and photos taken at intervals to document the visual variation in the surface as corrosion takes place. 


\section{Microstructure and Elemental Analysis}

It has been shown that pitting corrosion behaves differently for different intermetallics. FIG. 1(a). shows a polished substrate with two intermetallic particles circled and labelled 1 and 2. Utilizing EDS mapping these intermetallics have been identified as 1. CuMgAl (S-phase) and 2. CuFeMnAl. FIG. 1(b). identifies these same intermetallics after the surface was corroded for 4 hours in $0.5 \mathrm{M}$ $\mathrm{NaCl}$ solution and the corrosion product removed with $10 \% \mathrm{v} / \mathrm{v} \mathrm{HNO}_{3}$. This image depicts the two main types of pitting. In the case of the S-phase particle, intermetallic dissolution has dissolved the particle leaving a pit. The corrosion process for the $\mathrm{CuFeMnAl}$ particle has resulted in trenching, attacking the bulk around the intermetallic whilst leaving this inclusion relatively untouched.

An Environmental SEM (ESEM) will be used to provide high resolution images and elemental maps at various stages of the corrosion process. In particular, sites of pit initiation will be identified along with the proximity of intermetallics. It is believed that with the aid of an ESEM, this process of intermetallic dissolution and trenching can be observed in-situ. Particle Induced X-ray Emission (PIXE) analysis using a nuclear microprobe combined with phase correlation mapping will then be applied to investigate the association of particular intermetallics with the onset of deep pits in a corroded surface. The PIXE analysis provides detailed information on the types of intermetallics and the presence of trace elements, such as chlorine, which play an important role in the corrosion process.

\section{References}

[1] J.E. Hatch, American Society for Metals, Metals Park, OH (1984)

[2] L. Juffs, Masters Thesis, RMIT (2002)

[3] R. G. Buchheit et al., Electrochem. Soc. 144 (1997) 2621-2628.

[4] G.S. Wei, M. Gao and R.P. Wei, Corrosion (1996) 8

[5] Corrosion of Aluminium Alloys, ed J.R. Davis, ASM International (1999)

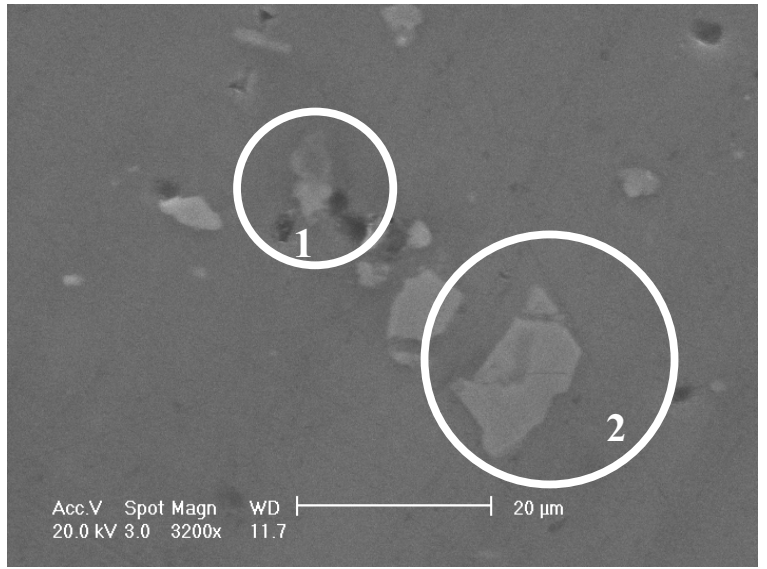

(a)

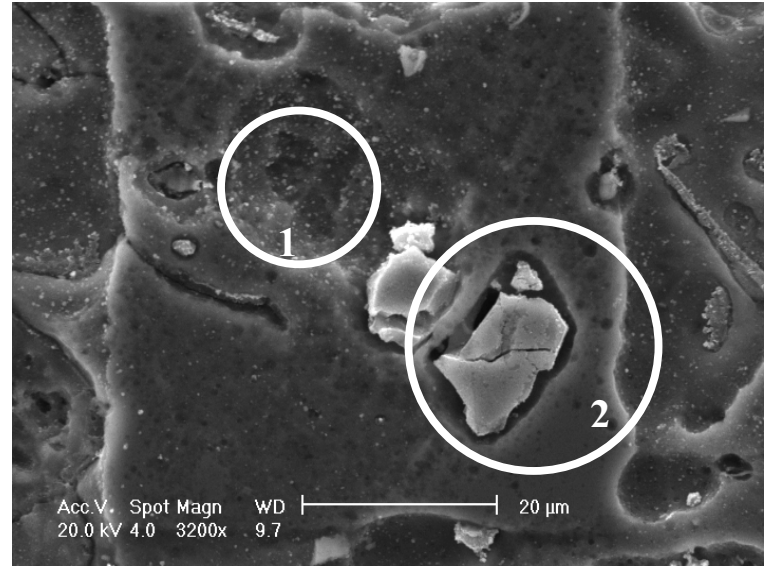

(b)

FIG. 1. SEM micrograph of a (a) polished and (b) corroded surface of AA2024-T3 